mimicry in the sexually deceptive pollination system therefore also provided an economical way for the plant to ensure its transfer of gametes.

Florian P. Schiestl ${ }^{\star}$, Manfred Ayasse ${ }^{\star}$, Hannes F. Paulus ${ }^{\star}$, Christer Löfstedt $\dagger$, Bill S. Hansson $\dagger$, Fernando Ibarra $\ddagger$, Wittko Francke

${ }^{\star}$ Institute of Zoology,

Department of Evolutionary Biology,

Althanstrasse 14, A-1090 Vienna, Austria

e-mail (F.P.S.):a8917722@unet.univie.ac.at

$\dagger$ Department of Ecology, Lund University,

S-22362 Lund, Sweden

$\$$ Department of Organic Chemistry,

University of Hamburg,

20146 Hamburg, Germany

1. Pouyanne, A. Bull. Soc. Hist. Nat. Afr. Noire 8, 6-7 (1917).

2. Kullenberg, B. Studies in Ophrys Pollination (Almquist \& Wiksells, Uppsala, 1961)

3. Paulus, H. F. \& Gack, C. Isr. J. Bot. 39, 43-79 (1990).

4. Linnaeus, C. Öländska och Gothländska Resa (Stockholm, 1745)

5. Borg-Karlson, A.-K. Phytochemistry 29, 1359-1387 (1990).

6. Bergström, G. in Biochemical Aspects of Plant and Animal

Coevolution (ed. Harborne, G.) 207-230 (Academic, New York, 1978).

Borg-Karlson, A.-K. \& Tengö, J. J. Chem. Ecol. 12, 1927-1941 (1986)

8. Baker, E. A. in The Plant Cuticle (eds Cutler, D. F., Alvin, K. L. \& Price, C. E.) 139-165 (Academic, London, 1982).

9. Nilsson, L. A. Trends Ecol. Evol. 7, 255-259 (1992).

10. Vogel, S. Akad. Wiss. Lit. (Mainz) Abh. Math.-Nat. Kl. 10, 1-165 (1962)

11. Schiestl, F. P., Ayasse, M., Paulus, H. F., Erdmann, D. \& Francke, W. J. Chem. Ecol. 23, 2881-2895 (1997).

\section{Fractal analysis of Pollock's drip paintings}

Scientific objectivity proves to be an essential tool for determining the fundamental content of the abstract paintings produced by Jackson Pollock in the late 1940s. Pollock dripped paint from a can onto vast canvases rolled out across the floor of his barn. Although this unorthodox technique has

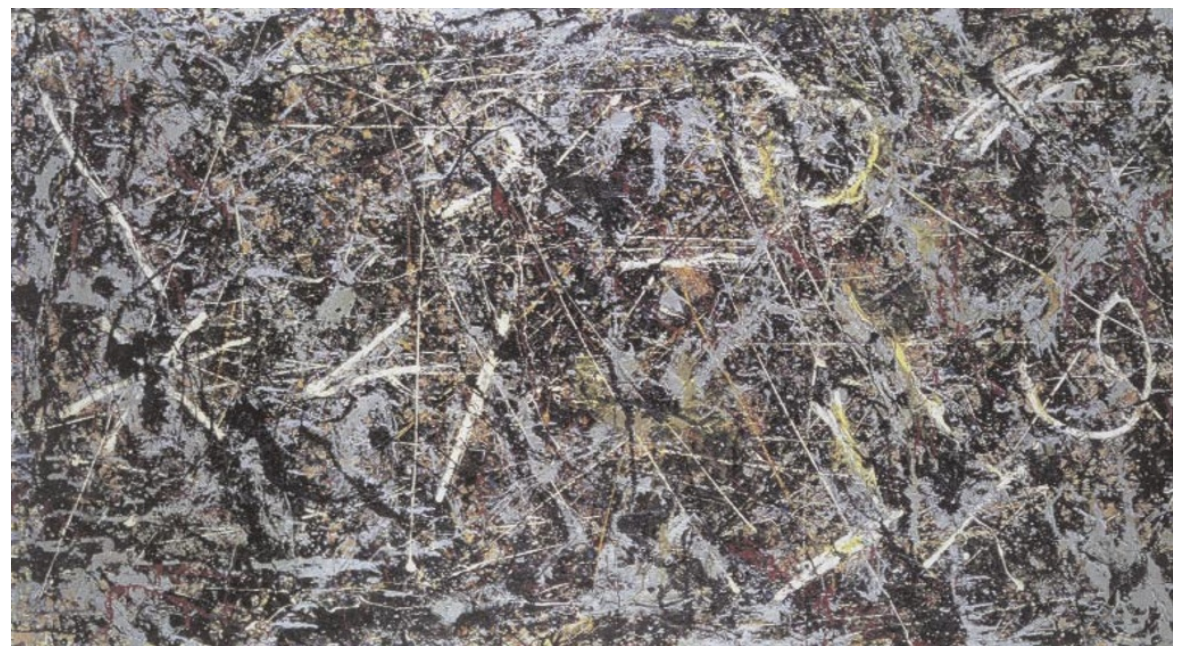

Figure 1 Alchemy, painted by Jackson Pollock in 1947. Drip paintings of this period are characterized by fractal dimensions close to 1.5. Reproduced by permission of ARS, NY and DACS, London, 1999.

been recognized as a crucial advancement in the evolution of modern art, the precise quality and significance of the patterns created are controversial. Here we describe an analysis of Pollock's patterns which shows, first, that they are fractal ${ }^{1}$, reflecting the fingerprint of nature, and, second, that the fractal dimensions increased during Pollock's career.

To quantify the fractal content of Pollock's drip paintings, such as Alchemy (Fig. 1), we used the well-established 'boxcounting' method $^{2}$ to calculate the fractal dimension $D$. We cover the scanned photograph of a Pollock painting with a computergenerated mesh of identical squares. The number of squares $N(L)$ that contain part of the painted pattern is then counted; this is repeated as the size, $L$, of the squares in the mesh is reduced. The largest size of square is chosen to match the canvas size $(L \approx 2.5 \mathrm{~m})$ and the smallest is chosen to match the finest paintwork ( $L \approx 1 \mathrm{~mm})$. For fractal behaviour, $N(L)$ scales according to $N(L) \propto L^{-D}$, where $1<D<2$. The $D$ values are extracted from the gradient of a graph of $\log N(L)$ plotted against $\log L$. This fractal analysis reveals two distinct $D$ values occurring over the ranges $1 \mathrm{~mm}<L<5 \mathrm{~cm}$ and $5 \mathrm{~cm}<\mathrm{L}<2.5 \mathrm{~m}$. Our analysis of a film of Pollock while painting shows that the fractal patterns occurring over the lower range are determined by the dripping process, whereas the fractal patterns across the higher range are shaped by his motions around the canvas.

Our analysis shows that Pollock refined his dripping technique: the fractal dimensions increased steadily through the years, from close to 1 in 1943 to 1.72 in 1952. Because $D$ follows such a distinct evolution with time, the fractal analysis could be used as a quantitative, objective technique both to validate and date Pollock's drip paintings. The change in $D$ reflects a dramatic evolution in visual character. His initial drip paintings of 1943 consisted of a single layer of paint trajectories that occupied only $20 \%$ of the $0.35-\mathrm{m}^{2}$ canvas area; by 1952 he was painting multiple layers of trajectories that covered over $90 \%$ of his $9.96-\mathrm{m}^{2}$ canvas. It is important that Pollock introduced his fractals systematically: the initial fractal layer essentially determined $D$ by acting as an anchor layer for the subsequent fractal layers, which then fine-tuned the value of $D$. Richard P. Taylor, Adam P. Micolich,

\section{David Jonas}

School of Physics, University of New South Wales, Sydney 2052, Australia

e-mail:rpt@newt.phys.unsw.edu.au

1. Mandelbrot, B. B. The Fractal Geometry of Nature (Freeman, New York, 1977).

2. Ott, E. Chaos in Dynamical Systems (Cambridge Univ. Press, 1993)

\section{Release from inhibition reveals the visual past}

Prolonged viewing of a high-contrast repetitive pattern such as a grating leads to adaptation of the corresponding visualprocessing channels ${ }^{1}$. We have found that such viewing also leads to the short-term establishment of a subthreshold trace in the brain that can cause a visual illusion of the pattern during rebound from the crossorientation inhibition ${ }^{2-4}$ that is induced by viewing moving patterns with an orthogonal orientation.

McKay ${ }^{5}$ reported that viewing a stationary pattern of radial lines causes the illusion of fine grains or wavy lines moving in circles. This has been explained as being due to antagonism between pattern and movement channels within an orientation column ${ }^{6}$. We have discovered a new visual after-effect by extending this model to explore antagonism between different orientation columns.

With the subject fixating at the centre, a windmill pattern is presented that slowly rotates at $0.2 \mathrm{~Hz}$ in one direction for $5 \mathrm{~s}$, and then in the other direction (Fig. 1a). If this is followed by a pattern orthogonal to the windmill, namely concentric rings, diverging and converging for $5 \mathrm{~s}$ each at $2 \mathrm{~Hz}$, viewing a blank screen after the concentric rings causes a vivid after-effect of a stationary windmill for a few seconds. This after-effect is quite different from that seen after viewing the concentric rings alone at $2 \mathrm{~Hz}$, if indeed any after-effect is visible. With a cyclical presentation of the sequence shown in Fig. 1a, a striking windmill-like after-effect is always seen after the concentric rings (bottom left blank in Fig. 1a), but no comparable after-effect is visible immediately after the windmill (top right blank in Fig. 1a). We found that the after-effect could be produced if the rings were delayed by up to $30 \mathrm{~s}$, but not after $60 \mathrm{~s}$. 


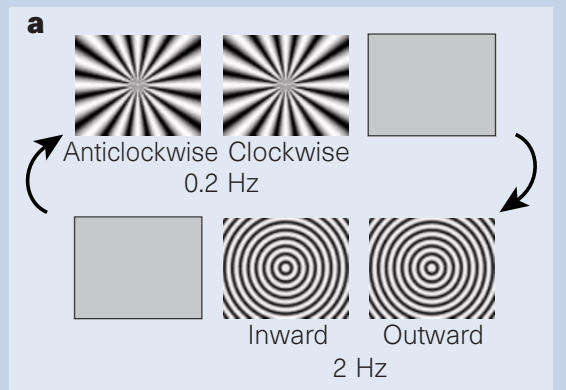

\section{c}
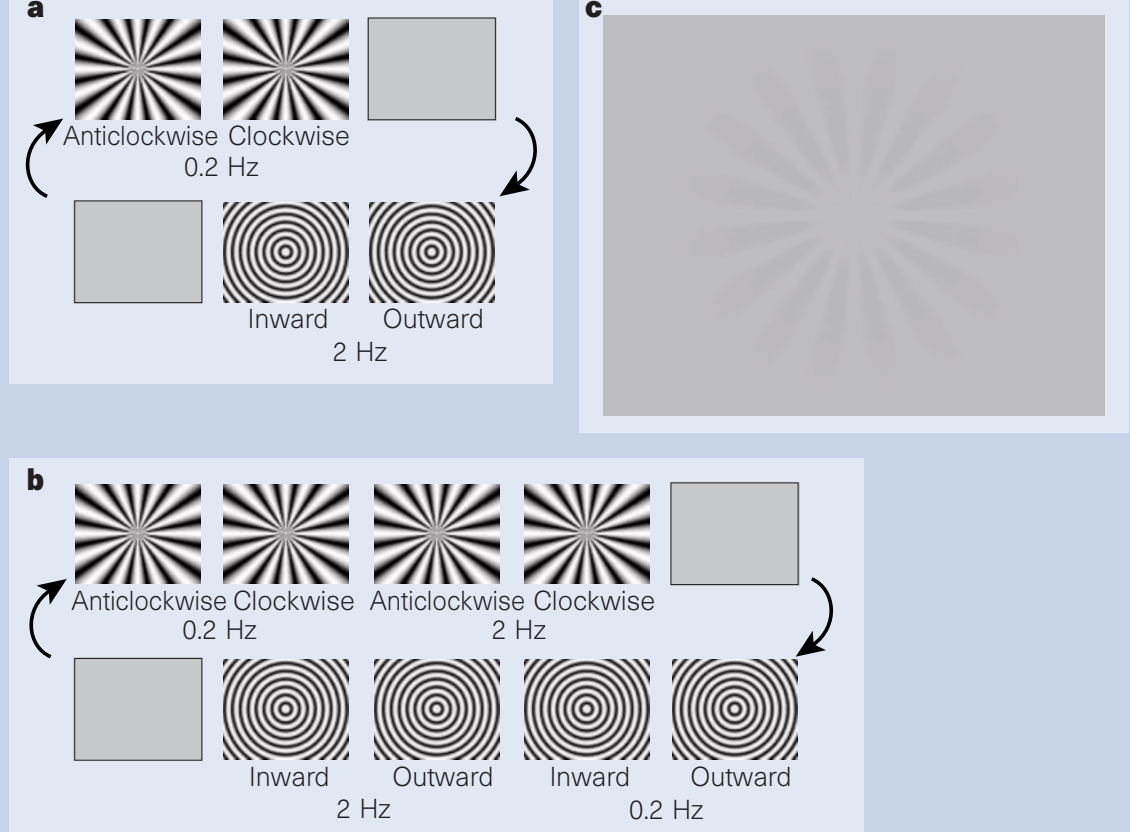

Figure 1 The stimuli used and the after-effect. $\mathbf{a}, \mathbf{b}$, Two different sequences of images that were used to test for the after-effect. The stimuli were produced with a VSG series 3 stimulus generator (Cambridge Research System, Rochester, UK). The radial patterns rotate clockwise or anticlockwise and the concentric rings move either inwards or outwards. The grey image is the blank screen of the same average luminance $\left(31.3 \mathrm{~cd} \mathrm{~m}^{-2}\right)$ as the patterns. The screen was placed at a distance of $85 \mathrm{~cm}$. c, A simulated enlarged image of the windmill after-effect that most observers see (lower left blank in $\mathbf{a}$ and $\mathbf{b}$ ). The contrast approximates to the strength of the percept. The Supplementary Information includes a demonstration of the illusions as in $\mathbf{a}$ and $\mathbf{b}$, as well as the script for viewing them on the VSG system.

The after-effect in Fig. 1a resembles the windmill pattern and is not related to the spatial frequency of the rings. If the angular frequency of the windmill changes, so does that of the after-effect. An essential requirement for the after-effect we describe is that the windmill pattern should be moving slowly, at $1 \mathrm{~Hz}$ or less, indicating that the stimulation of sustained pattern channels ${ }^{7}$ is crucial to the illusion.

The version shown in Fig. $1 \mathrm{~b}$ demonstrates the effect more vividly. If the windmill and concentric patterns are both presented at a slow and then at a faster rate (for example, $0.2 \mathrm{~Hz}$ and then $2 \mathrm{~Hz}$ ) with intervening blank screens, and the routine is cycled, after-effects can be seen in each blank period. In the period after the windmill (Fig. 1b, top right), a concentric ring after-effect can be seen; in the period after the concentric rings (bottom left), a windmill after-effect is seen. We presented these patterns to 13 naive subjects, who all reported seeing the illusions. Figure 1c approximates the windmill after-effect that most subjects perceive. It is unlikely that a retinal after-image could produce the illusion because the inducing patterns were not stationary and the after-effects seen were not of the immediately preceding patterns.

Even though the effects could be obtained with simple sine-wave gratings, we recommend radial patterns to avoid retinal after-images from pursuit eye movements. The after-effect is also stronger with radial patterns, which might be related to the meridional bias reported at many levels of the visual system ${ }^{4,8,9}$.

If, after the windmill pattern, a moving grating is presented instead of concentric rings, the after-effect is seen in an hourglass fashion, with the radial lines only at orientations orthogonal to the grating. This orientation selectivity, together with our finding that the effects are seen better binocularly than monocularly, suggests that a cortical locus is involved.

We propose the occurrence of two neuronal processes to explain the illusion. First, the stimulation of pattern channels not only leads to a decrease in sensitivity for that pattern $^{1}$, but also causes the deposition of a trace lasting for about half a minute, possibly at the synaptic level, akin to some form of short-term plasticity ${ }^{10}$. Second, this trace is brought to perceptual threshold by a rebound from inhibition caused by orthogonal orientations.

Georgeson ${ }^{6}$ explained McKay's rays by proposing that there is mutual antagonism between pattern and movement channels within the same orientation column. We suggest that this antagonism between pattern and movement channels also exists between orthogonal orientation columns.
The phenomenon that we have observed might serve as a strategy of the visual system for preventing the spurious excitation of contour detectors. Visual cortical cells, although best stimulated by bars moving perpendicular to the long axis of their receptive fields, often respond to small stimuli moving along this axis ${ }^{11}$. During eye movements, texture elements in the scene could therefore inappropriately stimulate cells whose optimum orientation is the same as the direction of movement. We propose that this excitation is prevented by inhibition from cells tuned to orthogonal orientations when the latter are activated by moving stimuli.

The transient existence of a stimulus trace is hard to explain. It might be an inevitable consequence of synaptic processes underlying short-term or long-term potentiation. It might also be a way of rapidly facilitating the perception of preexisting contours after an eye movement.

T. R. Vidyasagar ${ }^{\star}$, P. Buzás $\dagger$,

Z. F. Kisvárday $\dagger$, U. T. Eysel $\dagger$

${ }^{*}$ Psychobiology Laboratory, Division of Psychology, Australian National University,

Canberra, ACT 0200, Australia

$\dagger$ Department of Neurophysiology,

Faculty of Medicine, Ruhr-University Bochum,

44780 Bochum, Germany

e-mail:sagar@anu.edu.au

1. Blakemore, C. \& Campbell, F. W. J. Physiol., Lond. 203, 237-260 (1969).

2. Creutzfeldt, O. D., Kuhnt, U. \& Benevento, L. A. Exp. Brain Res. 21, 251-274 (1974).

3. Sillito, A. M. J. Physiol., Lond. 250, 305-329 (1975).

4. Vidyasagar, T. R., Pei, X. \& Volgushev, M. Trends Neurosci. 19, 272-277 (1996).

5. McKay, D. M. Nature 180, 849-850 (1957).

6. Georgeson, M. Nature 259, 413-415 (1976).

7. Kulikowski, J. J. \& Tolhurst, D. J. J. Physiol., Lond. 232, 149-162 (1973).

8. Levick, W. R. \& Thibos, L. N. J. Physiol., Lond. 329, 243-261 (1982).

9. Shou, T. \& Leventhal, A. G. J. Neurosci. 9, 4287-4302 (1989).

10. Fisher, S. A., Fischer, T. M. \& Carew, T. J. Trends Neurosci. 20, 170-177 (1997).

11. Wörgötter, F. \& Eysel, U. T. Exp. Brain Res. 76, 307-314 (1989). Supplementary information is available on Nature's World-Wide Web site (http://www.nature.com) or as paper copy from the London editorial office of Nature.

\section{Climate change related to egg-laying trends}

Analysis of 20 species of UK breeding birds over a 25-year period found a long-term trend towards earlier egg-laying ${ }^{1}$. Further studies have correlated such trends with spring temperatures (one species) ${ }^{2}$ or the North Atlantic Oscillation (three species) ${ }^{3}$. We have studied a data set spanning 57 years and find that laying date is related to temperature or rainfall for 31 of 36 species $(86 \%)$, and that $53 \%$ of species show longterm trends in laying date over time, of which $37 \%$ can be statistically accounted 